\title{
Oligoastenoteratozoospermia e ipoposia: non solo varicocele
}

\author{
Daniele Gianfrilli ${ }^{1}$
}

Accettato: 24 giugno 2020 / Pubblicato online: 26 ottobre 2020

(C) The Author(s) 2020

\section{Commento a weekendo n. 97}

Il quiz 97 "sembrava un semplice varicocele, invece..." (http://societaitalianadiendocrinologia.it/public//pdf/quiz97. pdf), si riferiva al caso di un ragazzo di 18 anni con varicocele sinistro di III grado radiologico con ipoposia (volume dell'eiaculato $0,9 \mathrm{ml}$ ), $\mathrm{pH}$ acido e oligoastenoteratozoospermia (OAT) severa, con numero di spermatozoi $\leq 5 \times 10^{6} / \mathrm{ml}$, motilità $10 \%$ lineare veloce + lenta, forme tipiche $3 \%$, leucociti nella norma, senza anamnesi rilevante e con caratteri sessuali secondari normali per età. Veniva ripetuto lo spermiogramma che confermava il quadro di OAT. Alla domanda "Quali ulteriori esami richiedereste?" meno della metà dei solutori forniva la risposta 4 indicata come corretta: "esami ormonali (testosterone totale, LH, FSH), cariotipo, microdelezioni del cromosoma Y, ecografia prostatica e vescichette seminali transrettale, ricerca mutazioni associate a fibrosi cistica", mentre l'altra metà rispondeva includendo il test di frammentazione del DNA spermatico (fDNA) (risposte 1 e 3 ) e dosaggi di altri ormoni (Prolattina, inibina B, TSH - risposte 1 e 2).

Il quiz non era di facile soluzione e le risposte, tutte simili tra loro e tutte potenzialmente corrette a una prima e rapida lettura, potevano trarre in inganno il lettore. Dopo aver confermato l'OAT con un secondo spermiogramma, il successivo passo per un corretto inquadramento diagnostico è indubbiamente una valutazione ormonale. Le linee guida 2018 della European Academy of Andrology (EAA) raccomandano nei casi di OAT severa di richiedere in prima battuta testosterone totale, FSH e LH. La prolattina deve essere dosata

$凶$ D. Gianfrilli

daniele.gianfrilli@uniroma1.it

1 Dipartimento di Medicina Sperimentale, Sapienza Università di Roma, Roma, Italia soltanto in caso di sospetto di ipogonadismo ipogonadotropo [1], mentre l'inibina $\mathrm{B}$ non è raccomandata nei dosaggi di routine in prima battuta. Anche lo studio della funzione tiroidea non è raccomandato in prima linea, ma solo se vi è forte sospetto clinico, in quanto è stata riportata raramente un'associazione tra OAT e disfunzione tiroidea [1].

Parallelamente, è opportuna una ricerca di eventuali cause genetiche di OAT. Infatti il 10,8\% dei pazienti con meno di 1 milione di spermatozoi per eiaculato ha una causa genetica di infertilità [2]. L'analisi del cariotipo permette dunque di evidenziare la Sindrome di Klinefelter che è la più frequente causa genetica di infertilità [2], seguita dalle microdelezioni del cromosoma Y [3]. Pertanto, questi due esami genetici vengono raccomandati in tutti i pazienti con numero di spermatozoi $<5 \times 10^{6}$ [1]. Ad oggi, la Società Italiana di Andrologia e Medicina della Sessualità (SIAMS) e l'EAA non raccomandano il test di frammentazione del DNA di routine nell'iter diagnostico del paziente con OAT [4]. L'esame può invece trovare indicazione nello studio di pazienti con infertilità idiopatica in previsione di una procreazione medicalmente assistita, nei casi di aborti ricorrenti e in pazienti che presentino fattori di rischio associati a incremento dei livelli di fDNA. Infine, dato che nel nostro caso erano presenti ipoposia e un $\mathrm{pH}$ acido, è necessario indagare anche le possibili cause ostruttive di OAT. Per questo è corretto richiedere un'ecografia prostatica transrettale con studio delle vescichette seminali [1]. Successivamente all'ecografia prostatica, in caso di sospetta ostruzione del tratto genitale, può essere indagata la presenza di mutazioni del gene CFTR per escludere la Fibrosi Cistica [1]. Tale condizione genetica solo raramente può essere anche associata a forme non ostruttive di infertilità e a quadri di oligospermia severa [5].

Funding Note Open access funding provided by Università degli Studi di Roma La Sapienza within the CRUI-CARE Agreement. 
Conflitti di interesse L'autore Daniele Gianfrilli dichiara di non avere conflitti di interesse.

Consenso informato Lo studio presentato in questo articolo non ha richiesto sperimentazione umana.

Studi sugli animali L'autore di questo articolo non ha eseguito studi sugli animali.

Nota della casa editrice Springer Nature rimane neutrale in riguardo alle rivendicazioni giurisdizionali nelle mappe pubblicate e nelle affiliazioni istituzionali.

Open Access This article is licensed under a Creative Commons Attribution 4.0 International License, which permits use, sharing, adaptation, distribution and reproduction in any medium or format, as long as you give appropriate credit to the original author(s) and the source, provide a link to the Creative Commons licence, and indicate if changes were made. The images or other third party material in this article are included in the article's Creative Commons licence, unless indicated otherwise in a credit line to the material. If material is not included in the article's Creative Commons licence and your intended use is not permitted by statutory regulation or exceeds the permitted use, you will need to obtain permission directly from the copyright holder. To view a copy of this licence, visit http://creativecommons.org/licenses/by/4.0/.

\section{Bibliografia}

1. Colpi GM, Francavilla S, Haidl G et al (2018) European Academy of Andrology guideline Management of oligo-asthenoteratozoospermia. Andrology 6(4):513-524

2. Punab M, Poolamets O, Paju P et al (2017) Causes of male infertility: a 9-year prospective monocentre study on 1737 patients with reduced total sperm counts. Hum Reprod 32(1):18-31

3. Krausz C, Hoefsloot L, Simoni M et al (2014) EAA/EMQN best practice guidelines for molecular diagnosis of Y-chromosomal microdeletions: state-of-the-art 2013. Andrology 2(1):5-19

4. Condorelli RA, Calogero AE (2018) Test della frammentazione del DNA spermatico: quando richiederlo e perché? Istruzioni per l'uso SIAMS. http://www.siams.info/wp-content/uploads/2018/ 06/25\%C2\%B0-28.06-Test-della-frammentazione-del-DNAspermatico-quando-richiederlo-e-perch\%C3\%A9.pdf. Accessed on $01 / 06 / 2020$

5. Yang L, Ren Z, Yang B et al (2020) The association between variants in the CFTR gene and nonobstructive male infertility: a meta-analysis. Andrologia 52(2):e13475 